\title{
JRRD $=$
}

\section{Effects of a flat prosthetic foot rocker section on balance and mobility}

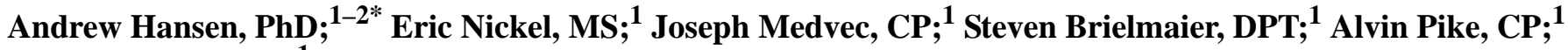 \\ Marilyn Weber, MD $^{1}$ \\ ${ }^{1}$ Minneapolis Department of Veterans Affairs Health Care System, Minneapolis, MN; ${ }^{2}$ Department of Physical Medi- \\ cine and Rehabilitation, Program in Rehabilitation Science, University of Minnesota, Minneapolis, MN
}

\begin{abstract}
Previous studies have shown that the effective rocker shape of the physiologic ankle-foot system during standing and fore-aft swaying is much flatter than that used during walking, which indicates a more stable base of support for the standing/ swaying activity. Previous work suggests that flat regions within the effective rocker shapes of prosthetic ankle-foot systems could provide enhanced stability for standing balance tasks. An experimental prosthetic foot was altered to provide three different flat region lengths within its effective rocker shape. It was hypothesized that longer flat regions of the effective rocker shape would lead to improved standing balance outcomes and reduced walking performance for unilateral transtibial prosthesis users. However, no significant changes were seen in the balance and mobility outcomes of 12 unilateral transtibial prosthesis users when using the three prosthetic foot conditions. Subjects in the study significantly preferred prosthetic feet with relatively low to moderate flat regions over those with long flat regions. All the subjects without loss of light touch or vibratory sensation selected the prosthetic foot with the shortest flat region. More work is needed to investigate the effects of prosthetic foot properties on balance and mobility of prosthesis users.
\end{abstract}

Key words: amputee, artificial limb, balance, foot, gait, mobility, prosthesis, prosthetic foot, standing, walking.

\section{INTRODUCTION}

Standing balance is maintained in nondisabled humans using three physiologic systems: visual, vestibular, and somatosensory [1]. A lower-limb amputation disturbs the somatosensory feedback system through loss of part of the proprioception by the physical removal of physiologic sensors. New feedback mechanisms for balance can be established through sensation of loads on the remaining residual limb (stump) tissues and the formation of an internal model of prosthesis behavior. However, in many cases, the sensation in the residual limb is also diminished or absent, complicating or preventing the development of new feedback mechanisms. Persons with unilateral amputations due to diabetes or vascular disease often also have a loss of sensation on the intact limb, further complicating their control of standing balance.

For many persons with lower-limb amputations, maintaining balance is challenging. Balance confidence is reduced in persons with lower-limb amputations. This level of confidence in balance correlates with measures of social activities and quality of life [2]. These findings suggest that interventions to improve balance may make lower-limb prosthesis users more confident in their balance, leading them to perform more social activities and have an enhanced quality of life.

Abbreviations: $\mathrm{ABC}=$ Activities-specific Balance Confidence Scale, EFLR = effective foot length ratio, $\mathrm{FL}=$ foot length, IRB = institutional review board, LCI-5 = Locomotor Capabilities Index5, LOS = limits of stability, LTEST $=$ L Test of Functional Mobility, MCT = motor control test, MFCL = Medicare Functional Classification Level, QS = quiet standing, STS = sit to stand, VA = Department of Veterans Affairs, WS = walking speed.

*Address all correspondence to Andrew Hansen, PhD; One Veterans Drive, Research-MS 151, Minneapolis, MN 55417; 612-467-2910; fax: 612-725-2093.

Email: andrew.hansen2@va.gov

http://dx.doi.org/10.1682/JRRD.2012.12.0229 
Falling is common in persons with lower-limb amputations [2]. Although the primary causes of falling in this population are not clear, interventions to improve balance during activities of daily life may reduce the occurrence of falls and fall-related healthcare costs.

Recent studies of the nondisabled physiologic anklefoot system during walking have shown that this system adapts to a variety of conditions (e.g., walking speed [WS], footwear, carriage of added weight) to maintain a curved effective rocker shape with a radius of approximately $1 / 3$ of the leg length [3-7]. Other work has shown that the physiologic ankle-foot system conforms to a much flatter effective rocker shape during standing and fore-aft swaying (radius 2 times leg length) [8]. The radii of the effective ankle-foot rocker shapes during walking and standing/ swaying are interesting when considered in a rocker inverted pendulum model of the human body, such as the one proposed by Gard and Childress [9]. In this model, a rocker with a radius of $1 / 3$ of the leg length is inherently unstable and naturally tends to fall over (Figure 1(a)). This characteristic supports the common description of walking as "falling from one foot to the other." For standing/swaying, a rocker with a radius of 2 times the leg length is mechanically stable and does not fall over, even with reasonable perturbations to the model (Figure 1(b)).

An analysis by Hansen and Wang found that a passive prosthetic ankle would require dramatically different torsional stiffness to mimic the effective rocker shapes achieved by neural control of the nondisabled ankle-foot system for walking and standing tasks [8]. This behavior would be difficult to achieve in a passive mechanical prosthesis. An alternative approach for passive mechanical ankle-foot prostheses is to conform to an effective shape that represents a compromise between biomimetic walking and standing shapes (Figure 1(c)). Curtze et al. found flattened middle sections of the effective rocker shapes of several commercially available prosthetic feet and suggested that this flat region could provide improved stability for standing [10].

Although it is known that some prosthetic feet have "flat regions" in their effective rocker shapes, there have been no investigations of the effect of the flat region length on balance and mobility. Understanding these effects could lead to improved prescription of prosthetic feet for persons with lower-limb amputations as well as design of improved ankle-foot prostheses. Thus, the purpose of this study was to examine the effect of prosthetic foot rocker flat region length on balance and mobility in

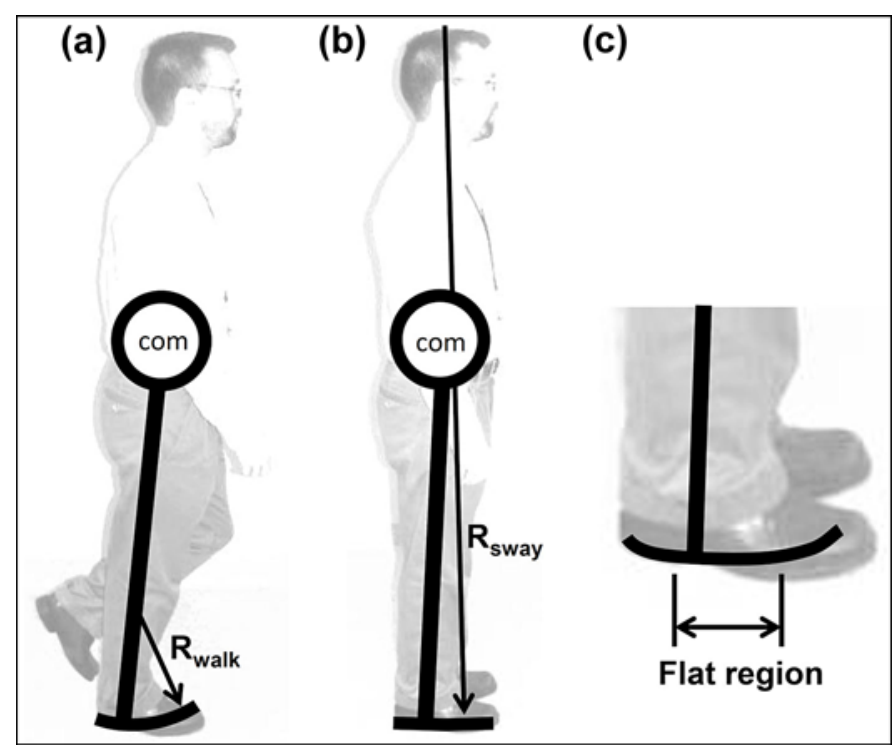

\section{Figure 1.}

Rocker inverted pendulum models of (a) walking, (b) standing, and (c) prosthetic foot representing compromise between biomimetic walking and standing/swaying shapes (this study). Radius of effective ankle-foot rocker shape for walking $\left(R_{\text {walk }}\right)$ is approximately $1 / 3$ of leg length, and radius for standing/swaying $\left(R_{\text {sway }}\right)$ is about 2 times leg length [8]. In this study, we examined prosthetic feet with effective rocker shapes having different flat regions representing compromises between biomimetic walking and standing/swaying shapes. com = center of mass.

unilateral transtibial prosthesis users. The study was achieved using an experimental prosthetic foot with the ability to easily alter the flat region length (prosthetic foot with an adjustable stability feature). We hypothesized that longer flat regions would result in improved standing balance performance and reduced walking performance of lower-limb prosthesis users. Standing balance was assessed by using static, volitional movement, and dynamic perturbation tests during standing. Walking mobility was assessed by using WS and a clinical test of functional mobility.

\section{METHODS}

\section{Experimental Prosthetic Foot Design}

The Shape\&Roll Prosthetic Foot, an experimental prosthetic foot described in earlier studies [11-14], was 
modified to simplify fabrication, accommodate a shoe heel height, and fit within a commercially available cosmetic foot shell (Figure 2). This modified Shape\&Roll Prosthetic Foot was fabricated from 1.5 in.-thick, 4 in.wide polypropylene bar stock purchased from McMasterCarr (part number 8782K77; Santa Fe Springs, California). The sagittal profile of the foot was cut with a band saw and the edges were sanded using a Trautman floor carver and a belt sander (Trautman Specialty Tools Inc; Minneapolis, Minnesota). A hole was drilled into the plastic and was counter-bored to accept an M10 bolt for connection to a standard solid ankle cushioned heel pyramid adapter. Cuts were placed in the forefoot of the plastic bar in the appropriate spacing that would mimic the roll-over shape achieved through neural control of the

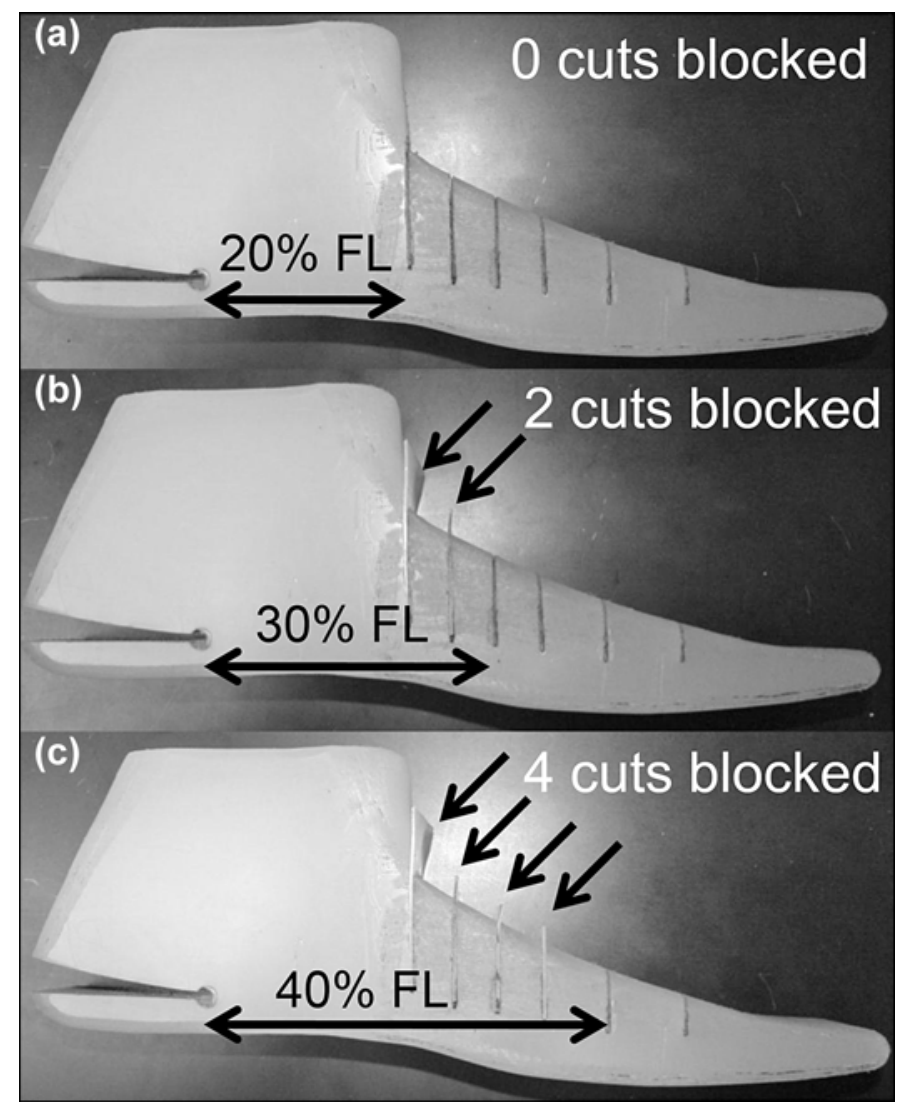

Figure 2.

Modified Shape\&Roll Prosthetic Foot with (a) 0, (b) 2, and (c) 4 forefoot cuts blocked. Blocking cuts prevents flexion of material under cuts and creates flat regions within effective rocker shape (Figure 1(c)). Cuts were blocked with $1 \mathrm{~mm}$-thick plastic sheets. $F L=$ foot length. physiologic ankle-foot system for walking (cut spacing determined by an iterative computer program [11]). The cuts were approximately $1 \mathrm{~mm}$ in thickness. To create an adjustable flat region foot, plastic pieces of approximately $1 \mathrm{~mm}$ in thickness were cut to fit within the forefoot cuts of the experimental foot. Placing pieces of plastic in the cuts leads to a longer flat region of the effective rocker shape by blocking flexion of the remaining material under the cuts, causing a stiffer forefoot. This method was used to alter the "stability" of the prosthetic foot.

For this study, we had prosthesis users test the experimental prosthetic foot in three conditions with approximate flat regions of 20,30, and 40 percent of foot length (FL) (respectively 0, 2, and 4 cuts blocked; Figure 2).To examine the differences in the effective rocker shapes of the experimental foot with these three conditions, we performed dynamic roll-over testing using pseudo-prostheses (stilts) as described previously in Hansen et al. [15] Testing occurred with the experimental foot conditions within an appropriately sized Aetrex Ambulator shoe (Teaneck, New Jersey).

\section{Subject Inclusion and Exclusion Criteria}

Subjects were recruited from the Regional Amputation Center at the Minneapolis Department of Veterans Affairs (VA) Health Care System. To be included in the study, subjects needed to have a unilateral transtibial amputation and be $18 \mathrm{yr}$ old or older. Subjects also needed to be able to understand the document for informed consent and be able to conduct multiple standing and swaying tasks and multiple mobility tests. Lastly, subjects needed to have a well-fitting endoskeletal prosthesis that could be disassembled and reassembled at the end of the study. Persons with skin breakdown on the residual limb, those who used exoskeletal prostheses, and those not otherwise meeting the inclusion criteria were excluded from the study.

\section{Power Analysis}

The number of subjects required to achieve statistical significance was determined based on the main variable of maximum excursion of the center of pressure in the direction of the prosthetic forefoot during the limits of stability (LOS) test. Type I error was set at 5 percent and type II error was set at 15 percent (i.e., a statistical power of 85\%). Using the iterative approach described by Lieber [16], and with the goal of being able to determine 
differences equal to one standard deviation of the measured value of maximum excursion of the center of pressure during the LOS test, we determined that 12 subjects were needed for the study.

\section{Description of Balance and Mobility Tests}

The following balance and mobility tests were used in the study to determine whether the prosthetic feet with longer flat regions would improve standing balance and reduce walking performance.

\section{Balance Tests}

Balance tests were conducted using a NeuroCom Smart EquiTest Clinical Research System (NeuroCom ${ }^{\circledR}$, Clackamas, Oregon). Although the Clinical Research System allows the investigator to program their own perturbation tests, we used the standard clinical tests provided by the system for this study to allow for comparison with previous and future studies. Tape was placed on the standing platform to indicate the positions of the subject's feet during the first standing balance test. For all other standing balance tests, the taped foot outlines were used to ensure consistent placement of the feet. Subjects wore shoes with their prostheses for all of the balance and mobility tests.

Limits of stability. Subjects shifted their weight in eight directions (forward-right, right, backward-right, backward, backward-left, left, forward-left, forward) within their functional base of support while having visual feedback of the net ground reaction force's center of pressure on a video screen. When prompted by a visual signal, the subjects would lean in the direction of the target and have $8 \mathrm{~s}$ to get their center of pressure as close to the target as possible. The primary outcome of interest was the maximum excursion of the center of pressure in the direction of the prosthetic forefoot. We expected that prosthetic feet with longer flat regions would lengthen the functional base of support in the direction of the prosthetic forefoot by providing enhanced stability (Figure 3). Subjects performed the LOS test one time for each foot condition.

Quiet standing. Subjects stood quietly on a force platform, first with eyes open and then with eyes closed (the first two conditions of the Sensory Organization Test). Each quiet standing (QS) trial (20 s in duration) was repeated twice for each foot condition. The equilibrium scores provided by the NeuroCom System were the outcome measures used to assess balance characteristics,

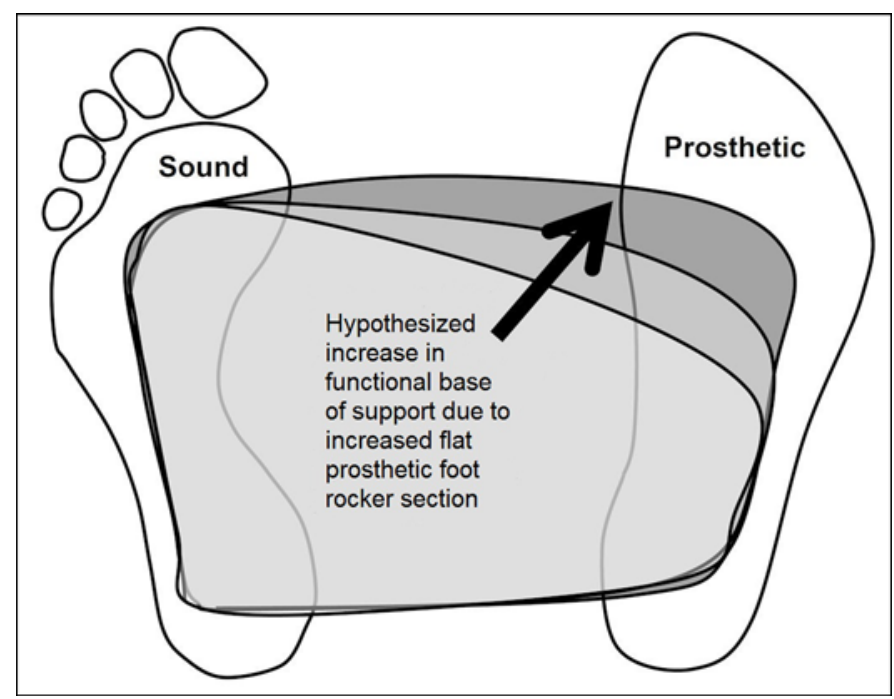

\section{Figure 3.}

Top-view drawing of feet of unilateral prosthesis user. Gray areas represent hypothetical functional bases of support, with darker gray areas representing larger bases of support. Hypothesis of study was that functional base of support would be increased with increasing flat prosthetic foot rocker sections, potentially leading to improved stability for prosthesis user.

with higher equilibrium scores suggesting improved standing balance outcomes.

Motor control test. Subjects stood quietly on a force platform with small, medium, and large translational perturbations to the platform introduced at unannounced times. The order of perturbations was small, medium, and large posterior translations of the platform followed by small, medium, and large anterior translations of the platform. The perturbations were achieved using a constant velocity of movement of the plate (i.e., a ramp function). The small, medium, and large perturbation amplitudes were $0.00694,0.01736$, and 0.03125 times the height of the subject, respectively. For a person that is 72 in. tall, for example, the small, medium, and large perturbation amplitudes were $0.5,1.25$, and 2.25 in., respectively. The small, medium, and large perturbations of the standing platform were conducted over durations of 250, 300, and $400 \mathrm{~ms}$, respectively. We were primarily interested in the number of stumbles that would occur during this testing that would require the subjects to take a step, use the overhead harness, or reach out and grab an upright structure with their hands. 


\section{Mobility Tests}

Sit to stand. This test monitored the time needed for subjects to go from a quiet sitting position to a QS position as well as the loading symmetry between sides during the transition. This test was administered three times per prosthetic foot condition using the NeuroCom Long Force Platform.

Walking speed. A stopwatch was used to determine the amount of time needed to traverse a $10 \mathrm{~m}$ distance down a hallway. The $10 \mathrm{~m}$ distance was divided by the time to calculate the WS. Subjects performed the WS test twice per prosthetic foot condition.

L Test of Functional Mobility. This test is defined as the time needed for a subject to go from sitting to standing, walk straight ahead for $3 \mathrm{~m}$, turn to the right and walk $7 \mathrm{~m}$, turn around and go back to the chair along the same "L" path, and sit back down onto the chair [17]. The L Test of Functional Mobility (LTEST) is a variant of the timed up and go test but was designed to be more relevant for lower-limb prosthesis users. Subjects performed the LTEST twice per prosthetic foot condition.

\section{Experimental Protocol}

Potential subjects first went through a consent process approved by the Minneapolis VA Institutional Review Board (IRB). The consent process culminated in written documentation of consent on IRB-approved forms. The height and weight of the subject were measured and recorded, as was the size of the prosthetic foot on his or her normal prosthesis. Relevant information about the normal prosthesis was also recorded, including prosthetic foot type, type of socket, and suspension method. Next, the subjects filled out a questionnaire called the Locomotor Capabilities Index-5 (LCI-5) [18], which describes their self-reported ability to move about their environment. Light touch and vibratory sensation of the subject's residual limb and remaining limb were measured next using the same sites and equipment as described in Quai et al. [19].

After initial tests establishing self-reported mobility and sensation, the subjects went through the series of balance (LOS, QS, motor control test [MCT]) and mobility (sit to stand [STS], WS, LTEST) tests with their normal prosthesis. Following the balance and mobility tests, the subjects filled out the Activities-specific Balance Confidence Scale $(\mathrm{ABC})$, a 16-point questionnaire gauging their confidence in their balance during a variety of activi- ties [20]. The primary objective of initially testing with the normal prosthesis was to gain baseline data to describe the subjects (e.g., WS and ABC), but also to allow the subjects to learn how to do the specific tests for the study (LOS, QS, MCT, STS, and LTEST).

After subjects completed the testing for their normal prosthesis, an experienced certified prosthetist disconnected the prosthetic socket from the rest of the prosthesis in a way that preserved the alignment (loosening two adjacent screws on the pyramid adapter) and connected the first experimental prosthetic foot and pylon. The length of the experimental foot was chosen to match the subject's usual prosthetic FL when possible. All changes to the prosthesis were performed by a certified prosthetist using standard clinical techniques. Balance (LOS, QS, MCT) and mobility (STS, WS, LTEST) testing were repeated for each of the three experimental foot conditions. Before testing on each foot, subjects walked up and down a $20 \mathrm{~m}$ hallway to briefly accommodate to the new foot condition.

The order of testing for the foot conditions was alternating, with odd-numbered participants testing the foot with the 40 percent FL flat region, then the 20 percent FL flat region and, lastly, the 30 percent FL flat region. Even-numbered participants tested the foot condition with the 20 percent FL flat region first, followed by the 40 percent FL flat region and the 30 percent FL flat region. This ordering was chosen to provide equal numbers of subjects using the extremes of foot conditions first in anticipation that some of the lowest-functioning prosthesis users would not be able to finish the study. Subjects and clinicians were blinded to the foot condition being tested. Only the engineers on the project were aware of the foot condition being tested.

After using the second and third experimental foot conditions, subjects were asked to provide a ranking describing their preference for the different foot conditions ( 1 = best foot, 2 = middle foot, and 3 = worst foot). In some cases, foot conditions were ranked as equal by the subjects. In those cases, the feet with equal rankings were given the same score but such that the sum of the three scores still equaled 6 (e.g., $1.5+1.5+3=6 ; 1+2.5+2.5=6$ ).

\section{Statistical Analysis}

We expected that longer flat regions would improve balance outcomes while reducing mobility outcomes. To determine whether statistical differences existed in the outcome measures as a function of the three foot conditions, a Friedman Test was used with a significance level 
set at $p<0.05$. When significant main effects were found, Wilcoxon signed-rank tests were used to determine significant differences between the foot conditions. The Bonferonni correction was used for multiple comparisons, adjusting the significance level for post hoc comparisons to $p<0.017$. The nonparametric test was chosen after discovering that 25 percent of the data sets were not normally distributed according to a KolmogorovSmirnov test of normality. SPSS (IBM Corporation; Armonk, New York) software was used to conduct all statistical tests.

\section{RESULTS}

The effective rocker shapes of the prosthetic foot conditions are shown in Figure $\mathbf{4}$ for qualitative comparison, including a magnified inset of the forefoot sections of the rocker shapes. Forward movement of the center of pressure (in the shank reference frame) versus shank angle plots for the foot conditions are shown in Figure 5. Curtze et al. used similar plots to determine a local radius of curvature of the effective rocker shape by examining the slope along the length of the curve (forward travel of

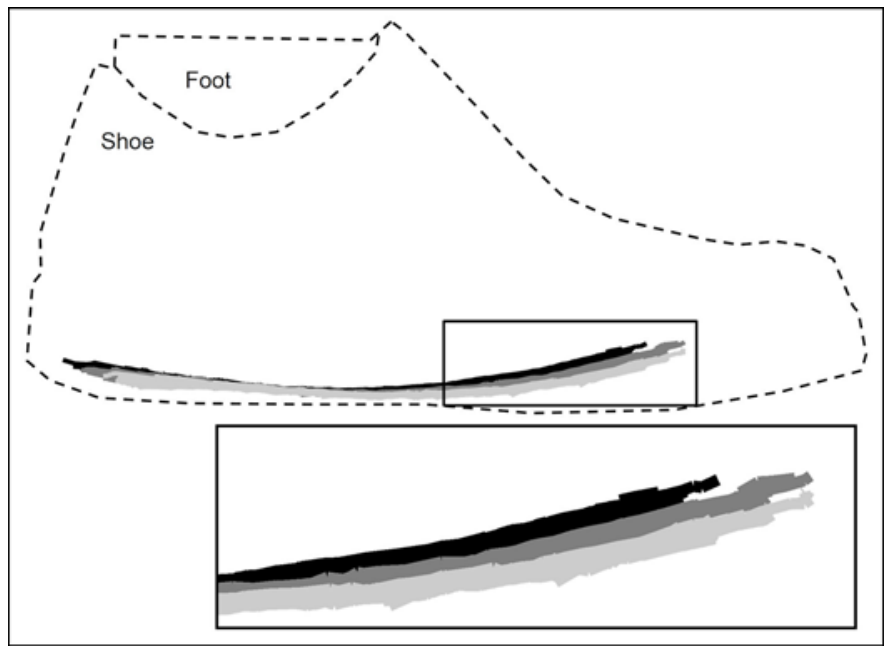

Figure 4.

Roll-over shape data for three prosthetic foot conditions (black = $20 \%$ foot length $[\mathrm{FL}]$ flat region, dark gray $=30 \% \mathrm{FL}$ flat region, light gray $=40 \% \mathrm{FL}$ flat region). Inset showing forefoot area in greater detail is shown at bottom. Outline of shoe and foot are for references purposes and are not necessarily to scale.

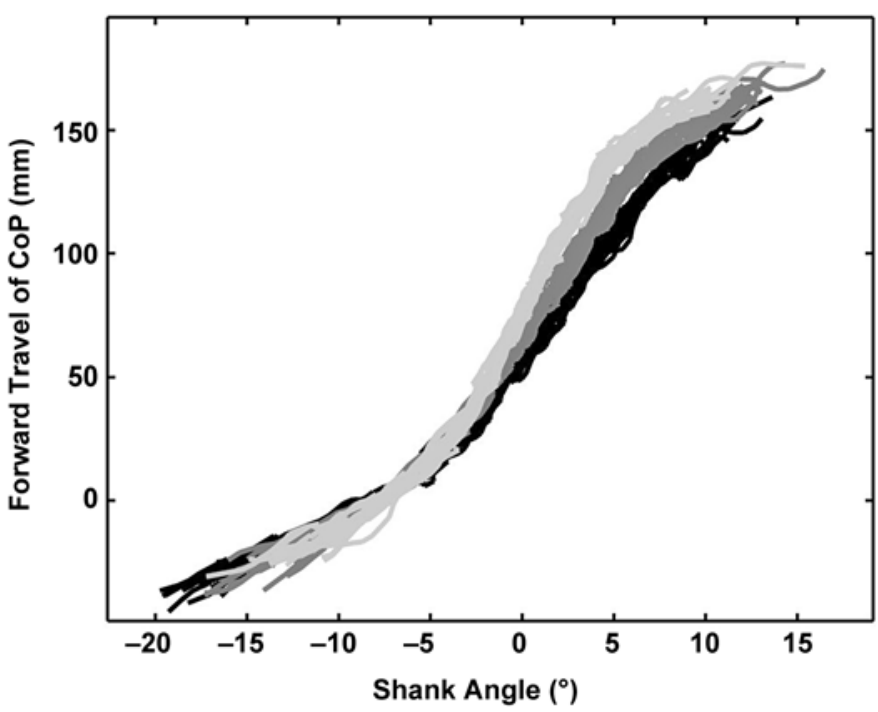

Figure 5.

Plot of forward travel of center of pressure (CoP) of ground reaction force versus shank angle for three foot conditions (black $=20 \%$ foot length $[\mathrm{FL}]$ flat region, dark gray $=30 \% \mathrm{FL}$ flat region, light gray $=40 \% \mathrm{FL}$ flat region). Slope of lines relates to instantaneous radius of curvature of effective rocker shapes [10]. Each line represents separate walking trial.

the center of pressure $\sim$ arc length $=$ radius $\times$ shank angle) [10]. Qualitatively, the slopes increase with longer flat regions (Figure 5), suggesting changes in the local radius of curvature as a function of the intervention.

Twelve subjects with unilateral transtibial amputations completed the study (Table 1 displays subject characteristics). One of the subjects had a transmetatarsal amputation on the contralateral side with a remaining FL of $16 \mathrm{~cm}$ (subject 2), and two subjects had amputations of the great toe on the contralateral side (subjects 6 and 11). For one subject, we used the closest available size of prosthetic foot $(28 \mathrm{~cm})$ to his usual prosthetic FL $(30 \mathrm{~cm})$. Baseline data collected for the research subjects (LCI-5, WS, ABC, and sensation) are shown in Table 2.

Balance outcomes were not significantly affected by the foot condition (Table 3). The maximum excursion in the direction of the prosthetic forefoot for the LOS test was not significantly affected by the foot condition ( $p=$ 0.19). Equilibrium scores during QS with eyes open and eyes closed were also not significantly affected by the foot condition ( $p=0.23$ and $p=0.32$, respectively). During the MCT testing, only one subject (subject 11) experienced a 
Table 1.

Characteristics of subjects in study and their usual prostheses.

\begin{tabular}{|c|c|c|c|c|c|c|c|c|}
\hline Subject & $\begin{array}{l}\text { Age } \\
(y r)\end{array}$ & $\begin{array}{c}\text { Height } \\
\text { (cm) }\end{array}$ & $\begin{array}{c}\text { Weight } \\
\text { (kg) }\end{array}$ & $\begin{array}{l}\text { Prosthetic Foot } \\
\text { Length } \\
\text { (cm) }\end{array}$ & $\begin{array}{c}\text { Cause of } \\
\text { Amputation }\end{array}$ & Suspension & Socket & Usual Prosthetic Foot \\
\hline 1 & 61 & 190 & 120 & 27 & PVD-diabetes & Sleeve & PTB & College Park Trustep \\
\hline 3 & 68 & 173 & 100 & 28 & PVD-diabetes & Sleeve & PTB & Otto Bock 1D10 \\
\hline 4 & 61 & 175 & 73 & 26 & Osteomyelitis & Pin liner & РTB & College Park Trustep \\
\hline 5 & 56 & 178 & 103 & 27 & Trauma & Pin liner & PTB & Ossur LP Variflex \\
\hline 7 & 64 & 175 & 84 & 25 & Trauma & Pin liner & РTB & Freedom Innovations Renegade LP \\
\hline $8^{\ddagger}$ & 48 & 188 & 121 & 30 & Trauma & Harmony & Other & Ossur Flexfoot Mod 3 \\
\hline 9 & 58 & 178 & 84 & 27 & Trauma & Pin liner & РTB & College Park Trustep \\
\hline 10 & 64 & 175 & 91 & 26 & Cancer & Pin liner & PTB & College Park Trustep \\
\hline $11^{\dagger}$ & 65 & 186 & 100 & 27 & PVD-diabetes & Sleeve & TSB & Freedom Innovations Renegade SX \\
\hline 12 & 78 & 172 & 97 & 26 & PVD-diabetes & Pin liner & PTB & Otto Bock C-Walk 1C40 \\
\hline
\end{tabular}

Table 2.

Baseline data for study subjects.

\begin{tabular}{|c|c|c|c|c|c|}
\hline Subject & $\begin{array}{l}\text { LCI-5 } \\
\text { Score }\end{array}$ & $\begin{array}{l}\text { ABC Composite } \\
\text { Score }\end{array}$ & $\begin{array}{l}\text { WS } \\
(\mathrm{m} / \mathrm{s})\end{array}$ & $\begin{array}{c}\text { Light Touch Sensation Loss } \\
\text { (No. of Sites) }\end{array}$ & $\begin{array}{c}\text { Vibration Sensation Loss } \\
\text { (No. of Sites) }\end{array}$ \\
\hline$\overline{1}$ & 54 & 84 & 1.29 & 9 & 4 \\
\hline 2 & 54 & 78 & 0.76 & $7^{*}$ & $1^{*}$ \\
\hline 3 & 50 & 95 & 1.05 & 8 & 4 \\
\hline 4 & 56 & 100 & 1.11 & 1 & 1 \\
\hline 5 & 56 & 70 & 1.55 & 1 & 0 \\
\hline 6 & 54 & 79 & 1.45 & $5^{*}$ & $2^{*}$ \\
\hline 7 & 56 & 90 & 1.18 & 0 & 0 \\
\hline 8 & 56 & 100 & 1.78 & 0 & 0 \\
\hline 9 & 56 & 89 & 1.45 & 0 & 0 \\
\hline 10 & 56 & 99 & 1.23 & 0 & 0 \\
\hline 11 & 55 & 83 & 1.26 & $9^{*}$ & $1^{*}$ \\
\hline 12 & 52 & 90 & 1.03 & 1 & 0 \\
\hline Average & 55 & 88 & 1.26 & - & - \\
\hline $\mathrm{SD}$ & 2 & 10 & 0.27 & - & - \\
\hline
\end{tabular}

loss of balance when using the prosthetic foot with the shortest flat region (20\% FL flat region) during the large forward movements of the force platform; subject 11 took small steps backward with the sound limb.

Mobility outcomes were also not significantly affected by the foot condition (Table 4). The prosthetic foot condi- tion did not significantly affect either the time needed to transition between quiet sitting to QS $(p=0.34)$ or the weight symmetry during the STS transition $(p=0.63)$. WS was not significantly affected by the foot condition ( $p=$ $0.20)$. The LTEST times were significantly affected by the foot condition ( $p=0.046)$. However, post hoc testing did 
JRRD, Volume 51, Number 1, 2014

not find significant differences between foot conditions for

Table 3.

Balance outcomes and preferences for different foot conditions (flat region lengths of $20 \%$, 30\%, and $40 \%$ foot length [FL]).

\begin{tabular}{|c|c|c|c|c|c|c|c|c|c|c|c|}
\hline \multirow[t]{2}{*}{ Subject } & \multicolumn{3}{|c|}{$\begin{array}{l}\begin{array}{c}\text { LOS Maximum } \\
\text { Excursion }\end{array} \\
\text { Flat Region Length }\end{array}$} & \multicolumn{3}{|c|}{$\begin{array}{c}\text { QS Equilibrium Score } \\
\text { (Eyes Open) } \\
\text { Flat Region Length }\end{array}$} & \multicolumn{3}{|c|}{$\begin{array}{c}\begin{array}{c}\text { QS Equilibrium Score } \\
\text { (Eyes Closed) }\end{array} \\
\text { Flat Region Length }\end{array}$} & \multirow[t]{2}{*}{$\begin{array}{l}\text { Most Preferred } \\
\text { Foot }^{*}\end{array}$} & \multirow[t]{2}{*}{$\begin{array}{c}\text { Least Preferred } \\
\text { Foot }^{*}\end{array}$} \\
\hline & 20 & 30 & 40 & 20 & 30 & 40 & 20 & 30 & 40 & & \\
\hline 1 & 81 & 78 & 86 & 91 & 90 & 90 & NA & NA & NA & $20 \% \mathrm{FL} / 30 \% \mathrm{FL}$ & $40 \% \mathrm{FL}$ \\
\hline 2 & 67 & 61 & 52 & 95 & 95.5 & 94 & 84 & 89.5 & 89 & $30 \% \mathrm{FL}$ & $40 \% \mathrm{FL}$ \\
\hline 3 & 77 & 71 & 77 & 92.5 & 95 & 95 & 92 & 95 & 92.5 & $30 \% \mathrm{FL}$ & $40 \% \mathrm{FL}$ \\
\hline 4 & 91 & 102 & 96 & 96.5 & 96 & 96.5 & 94 & 94.5 & 91 & $30 \%$ FL & $40 \% \mathrm{FL}$ \\
\hline 5 & 117 & 98 & 104 & 92 & 92 & 95 & 88.5 & 90 & 90 & $40 \% \mathrm{FL}$ & $20 \% \mathrm{FL}$ \\
\hline 6 & 81 & 59 & 66 & 88.5 & 88 & 89 & 86 & 84 & 85.5 & $30 \%$ FL & $40 \% \mathrm{FL}$ \\
\hline 7 & 74 & 66 & 70 & 96.5 & 95.5 & 97 & 91 & 91.5 & 95 & $20 \% \mathrm{FL}$ & $40 \% \mathrm{FL}$ \\
\hline 8 & 83 & 85 & 87 & 95 & 95.5 & 94 & 92.5 & 85 & 91.5 & $20 \% \mathrm{FL} / 30 \% \mathrm{FL}$ & $40 \% \mathrm{FL}$ \\
\hline 9 & 85 & 89 & 87 & 93.5 & 94 & 94 & 92 & 89.5 & 92.5 & $20 \% \mathrm{FL}$ & $40 \% \mathrm{FL}$ \\
\hline 10 & 97 & 83 & 92 & 95 & 96 & 96 & 86.5 & 91.2 & 93 & $20 \% \mathrm{FL}$ & $40 \% \mathrm{FL}$ \\
\hline 11 & 70 & 68 & 76 & 93.5 & 90.5 & 96 & 88 & 89 & 84.5 & $20 \% \mathrm{FL}$ & $30 \% \mathrm{FL} / 40 \% \mathrm{FL}$ \\
\hline 12 & 80 & 68 & 62 & 90 & 86 & 92 & 80 & 88.5 & 91.5 & $30 \% \mathrm{FL}$ & $40 \% \mathrm{FL}$ \\
\hline$\overline{\text { Median }}$ & 81.0 & 74.5 & 81.5 & 93.5 & 94.5 & 94.5 & 88.5 & 89.5 & 91.5 & - & - \\
\hline$p$-Value & 0.19 & - & - & 0.23 & - & - & 0.32 & - & - & - & - \\
\hline
\end{tabular}

Table 4.

Mobility outcomes for different foot conditions (flat region lengths of 20\%, 30\%, and $40 \%$ foot length).

\begin{tabular}{|c|c|c|c|c|c|c|c|c|c|c|c|c|}
\hline \multirow{3}{*}{ Subject } & \multirow{2}{*}{\multicolumn{3}{|c|}{$\begin{array}{c}\text { STS Loading Symmetry* } \\
(\%) \\
\text { Flat Region Length }\end{array}$}} & \multirow{2}{*}{\multicolumn{3}{|c|}{$\begin{array}{c}\begin{array}{c}\text { STS Weight Transfer } \\
\text { Time (s) }\end{array} \\
\text { Flat Region Length }\end{array}$}} & \multirow{2}{*}{\multicolumn{3}{|c|}{$\begin{array}{c}\begin{array}{c}\text { WS } \\
(\mathrm{m} / \mathrm{s})\end{array} \\
\text { Flat Reoion Lenoth }\end{array}$}} & \multicolumn{3}{|c|}{$\begin{array}{l}\text { LTEST Time } \\
\text { (s) }\end{array}$} \\
\hline & & & & & & & & & & Flat & gion I & gth \\
\hline & 20 & 30 & 40 & 20 & 30 & 40 & 20 & 30 & 40 & 20 & 30 & 40 \\
\hline 1 & -23 & -11 & 2 & 0.24 & 0.24 & 0.2 & 1.51 & 1.49 & 1.43 & 18 & 18 & 20 \\
\hline 2 & -1 & -6 & -1 & 1.29 & 1.24 & 1.23 & 0.63 & 0.59 & 0.53 & 43 & 47 & 49 \\
\hline 3 & -44 & -37 & -48 & 1.53 & 0.27 & 0.35 & 1.08 & 1.04 & 1.06 & 24 & 24 & 23 \\
\hline 5 & -24 & -28 & -36 & 0.22 & 0.25 & 0.24 & 1.63 & 1.63 & 1.62 & 16 & 16 & 17 \\
\hline 6 & -8 & -20 & -16 & 0.3 & 0.25 & 0.31 & 1.47 & 1.55 & 1.53 & 23 & 22 & 22 \\
\hline 7 & -21 & -15 & -37 & 0.2 & 0.18 & 0.16 & 1.26 & 1.3 & 1.25 & 22 & 21 & 22 \\
\hline 8 & -11 & -2 & -6 & 0.29 & 0.26 & 0.27 & 1.76 & 1.84 & 1.95 & 13 & 11 & 12 \\
\hline 9 & 27 & -42 & -32 & 0.19 & 0.25 & 0.17 & 1.46 & 1.47 & 1.44 & 18 & 18 & 18 \\
\hline Median & -13.0 & -21.5 & -16.5 & 0.2 & 0.3 & 0.3 & 1.28 & 1.34 & 1.29 & 21.1 & 20.5 & 21.3 \\
\hline$p$-Value & 0.63 & - & - & 0.34 & - & - & 0.20 & - & - & 0.046 & - & - \\
\hline
\end{tabular}


LTEST times (20\% FL vs 30\% FL, $p=0.08 ; 20 \%$ FL vs $40 \%$ FL, $p=0.43 ; 30 \%$ FL vs $40 \%$ FL, $p=0.02$ ). Note that the difference in times for the LTEST was nearly significant between the 30\% FL and 40\% FL flat region conditions.

The preference ranking for the prosthetic feet was significantly affected by the foot condition ( $p=0.002$; Table 2). Post hoc testing showed a significant difference in preference ranking between the $30 \% \mathrm{FL}$ and $40 \% \mathrm{FL}$ flat region conditions ( $p=0.006)$, but not between the $20 \% \mathrm{FL}$ and the $30 \% \mathrm{FL}$ flat region conditions $(p=0.78)$ or between the $20 \%$ FL and $40 \%$ FL flat region conditions $(p=0.02)$. Note that the preference ranking between the 20\% FL and 40\% FL flat region conditions was nearly significant.

Some of the subjects offered additional comments on the different experimental feet. Subject 1 felt "more balanced" overall when using the foot with the shortest flat region compared with the foot with the longest flat region. Subject 2 stated that the foot with the longest flat region felt unstable for walking and had a "halting" action during walking and that the roll-over with this foot was not smooth. Subject 2 also mentioned that the foot with the longest flat region felt acceptable for standing. Other subjects also had similar comments regarding the foot with the longest flat region, indicating that it felt too rigid and did not provide a smooth roll-over during walking. Subject 5 was the only subject who preferred the foot with the longest flat region, indicating that he felt less pain in his hip during some of the testing (MCT, WS, and LTEST) when using the foot with the longest flat region compared with the foot with the shortest flat region.

\section{DISCUSSION}

Our hypothesis was that prosthetic feet with longer flat regions would improve standing balance outcomes and worsen walking performance. This hypothesis was not supported in our study for the foot conditions tested-no significant changes were found in standing balance or walking outcome measures. The changes in prosthetic foot conditions tested in this study were subtle (Figures 4 and 5) and perhaps not drastic enough to lead to balance and mobility changes. The subjects tested in this study may have been robust enough in their balance and mobility function to compensate for the small changes in foot conditions. Future studies using more pronounced differences in flat surface conditions may show the changes that were hypothesized in this study. Despite subtle changes in effective rocker characteristics, the subjects in the study were able to perceive differences between at least two of the three foot conditions, leading to significantly different rankings for the foot conditions. Most subjects (11 out of 12) disliked the prosthetic foot with the longest flat region, indicating that it was too rigid and did not provide a smooth roll-over. However, the subject with the lowest balance confidence score (subject 5) was the only subject in the study that preferred the prosthetic foot with the longest flat region. Subject 5's balance confidence score was 70, which is still above the average score (64.9) of balance confidence from a large sample of persons with transtibial amputations [21]. Future studies of prosthetic foot conditions with subjects with low balance confidence may provide further insight into this finding. Future studies should also characterize the usual prosthetic foot of the research subjects to see whether they tend to prefer feet most like their usual prosthetic foot.

The subjects in the study all had remaining control of the knee on the side of the amputation (i.e., their amputations were below the knee), and we observed that the subjects may have used a knee strategy to control their balance. More compliant feet can likely be accounted for with increased control of knee flexion during standing and leaning activities, within reasonable limits, although further studies would be needed to investigate this hypothesis. Further studies could also investigate the effects of the rocker's flat region on balance and mobility of transfemoral prosthesis users, who would not be able to actively control knee flexion on the prosthetic side during standing without losing stability.

In this study, we found that subjects with no loss of sensation $(n=4)$ tended to prefer the foot condition with the shortest flat region (20\% FL), while subjects with sensation loss $(n=8)$ tended to prefer the foot with a slightly longer flat region ( $30 \%$ FL). However, some deviations from this tendency occurred (e.g., subject 11 preferred the foot with $20 \%$ FL flat region despite having many sites with sensation loss). Subjects without sensation loss may be able to more effectively use the proprioception information from the socket interface (i.e., pressures between the socket and the residual limb tissues) during standing tasks, allowing them to balance more effectively with a more flexible prosthetic foot compared with persons with sensation loss. Further testing is needed to explore this finding. 
Previous studies using the Shape\&Roll Prosthetic Foot have determined that both shorter effective keel lengths and overly compliant keels can lead to a "dropoff” effect during walking, which leads to a higher first peak in the ground reaction force on the sound limb [1213]. The experimental foot conditions used in this study did not include short or overly compliant keels. Instead, this study explored a smaller range of prosthetic foot properties, all with relatively long effective FLs. The effective foot length ratios (EFLRs) of the feet tested in this study ranged from 0.83 to 0.88 . Previous work of Hansen et al. showed a range of EFLRs between 0.6 and 0.8 for commercially available prosthetic feet made in the 1990s [22]. More recent testing in our laboratory suggests ranges of EFLRs for more modern feet between 0.66 and 0.93 (unpublished results). Further studies with prosthetic feet having shorter effective foot lengths might show reductions in balance properties, namely reduced functional bases of support in the direction of the prosthetic forefoot (Figure 3). Further investigation is needed because many prosthetic feet prescribed for $\mathrm{K} 1$ and $\mathrm{K} 2$ prosthesis users have short effective keel lengths.

The study was only powered to determine differences in the maximum excursion of the center of pressure in the direction of the prosthetic forefoot. We did not find a significant difference in this outcome, perhaps because of the reasons mentioned earlier (long EFLRs for all feet tested, observed knee flexion with use of more flexible feet). The other outcome measure data collected in this study can be used as pilot data for powering future studies. For example, using a larger sample size in a future study may be able to determine a significant difference in the LTEST for prosthetic feet with different flat region lengths.

One limitation of the study was that the 30 percent FL flat region condition was always tested last. As mentioned earlier, we chose to examine the extremes $(20 \%$ and $40 \%$ FL flat regions) in an alternating order in the thought that some prosthesis users would not be able to test all foot conditions in the same day. This limitation in order of testing may have biased the results for or against the foot with the 30 percent FL flat region. Another limitation of the study was that the accommodation time for each foot condition was brief. Perhaps if more time had been allotted to accommodate to each foot condition, the results would have been different. However, the advantage of testing multiple conditions on the same day is that other factors that vary from day to day (e.g., mood, level of fatigue) are reduced or eliminated from the study.
A further limitation of the study is the small sample size and the high functional levels of the subjects. It is possible that varying the flat region of prosthetic feet could be more useful in lower functioning prosthesis users (e.g., those in the $\mathrm{K} 1$ level). The number of tests involved in this study and the repeated testing of different foot conditions was not feasible for prosthesis users in the K1 Medicare Functional Classification Level (MFCL). In the future, further testing of prosthesis users in the K1 MFCL should be conducted using a reduced set of outcome measures. Varying the flat region of prosthetic feet could also be useful for prosthesis users with bilateral and/or higher level amputations (e.g., transfemoral amputations).

\section{CONCLUSIONS}

The Shape\&Roll Prosthetic Foot provides a simple and inexpensive approach for testing the effects of foot conditions on mobility and balance. Although measures such as the EFLR offer some basis for comparison, more work is needed to compare results of testing with experimental feet such as the Shape\&Roll Prosthetic Foot with results of testing commercially available prosthetic feet. Improving the understanding of prosthetic foot features and their effects on prosthesis users should improve our ability to prescribe effective components and lead to improved designs of lower-limb prostheses.

\section{ACKNOWLEDGMENTS}

\section{Author Contributions:}

Study concept and design: A. Hansen.

Acquisition of data: A. Hansen, E. Nickel, J. Medvec, S. Brielmaier, A. Pike.

Analysis and interpretation of data: A. Hansen, E. Nickel.

Drafting of manuscript: A. Hansen.

Critical revision of manuscript for important intellectual content:

A. Hansen, E. Nickel, J. Medvec, S. Brielmaier, A. Pike, M. Weber. Statistical analysis: A. Hansen.

Obtained funding: A. Hansen.

Administrative, technical, or material support: A. Hansen, E. Nickel, J. Medvec, S. Brielmaier, A. Pike, M. Weber.

Study supervision: A. Hansen.

Financial Disclosures: The authors have declared that no competing interests exist.

Funding/Support: This material was based on work supported by the VA, Veterans Health Administration, Rehabilitation Research and Development Service (grant A6567R to Dr. Hansen). 
Additional Contributions: The authors also acknowledge Peggy Merchak, MA, OTR/L, CHT, for her assistance in recruitment of research subjects for the study.

Institutional Review: Potential subjects first went through a consent process approved by the Minneapolis VA IRB. The consent process culminated in written documentation of consent on IRB-approved forms.

Participant Follow-Up: The authors do not plan to inform participants of the publication of this study.

\section{REFERENCES}

1. Winter DA. Anatomy, biomechanics, and control of balance during standing and walking. Kitchener, Ontario (Canada): Waterloo Biomechanics; 1995.

2. Miller WC, Deathe AB, Speechley M, Koval J. The influence of falling, fear of falling, and balance confidence on prosthetic mobility and social activity among individuals with a lower extremity amputation. Arch Phys Med Rehabil. 2001;82(9):1238-44. [PMID:11552197] http://dx.doi.org/10.1053/apmr.2001.25079

3. Hansen AH, Childress DS, Knox EH. Roll-over shapes of human locomotor systems: Effects of walking speed. Clin Biomech (Bristol, Avon). 2004;19(4):407-14. [PMID:15109762] http://dx.doi.org/10.1016/j.clinbiomech.2003.12.001

4. Hansen AH, Childress DS. Effects of shoe heel height on biologic rollover characteristics during walking. J Rehabil Res Dev. 2004;41(4):547-54. [PMID:15558383] http://dx.doi.org/10.1682/JRRD.2003.06.0098

5. Hansen AH, Childress DS. Effects of adding weight to the torso on roll-over characteristics of walking. J Rehabil Res Dev. 2005;42(3):381-90. [PMID:16187250] http://dx.doi.org/10.1682/JRRD.2004.04.0048

6. Wang CC, Hansen AH. Response of able-bodied persons to changes in shoe rocker radius during walking: Changes in ankle kinematics to maintain a consistent roll-over shape. J Biomech. 2010;43(12):2288-93. [PMID:20483413] http://dx.doi.org/10.1016/j.jbiomech.2010.04.036

7. Hansen AH, Childress DS. Investigations of roll-over shape: Implications for design, alignment, and evaluation of ankle-foot prostheses and orthoses. Disabil Rehabil. 2010;32(26):2201-9. [PMID:20626257] http://dx.doi.org/10.3109/09638288.2010.502586

8. Hansen AH, Wang CC. Effective rocker shapes used by able-bodied persons for walking and fore-aft swaying: Implications for design of ankle-foot prostheses. Gait Posture. 2010;32(2):181-84. [PMID:20471833] http://dx.doi.org/10.1016/j.gaitpost.2010.04.014

9. Gard SA, Childress DS. What determines the vertical displacement of the body during normal walking? J Prosthet
Orthot. 2001;13:64-67.

http://dx.doi.org/10.1097/00008526-200109000-00009

10. Curtze C, Hof AL, van Keeken HG, Halbertsma JP, Postema K, Otten B. Comparative roll-over analysis of prosthetic feet. J Biomech. 2009;42(11):1746-53.

[PMID:19446814]

http://dx.doi.org/10.1016/j.jbiomech.2009.04.009

11. Sam M, Childress DS, Hansen AH, Meier MR, Lambla S, Grahn EC, Rolock JS. The 'shape\&roll' prosthetic foot: I. Design and development of appropriate technology for lowincome countries. Med Confl Surviv. 2004;20(4):294-306. [PMID:15688881] http://dx.doi.org/10.1080/1362369042000285937

12. Hansen AH, Meier MR, Sessoms PH, Childress DS. The effects of prosthetic foot roll-over shape arc length on the gait of trans-tibial prosthesis users. Prosthet Orthot Int. 2006;30(3):286-99. [PMID:17162519] http://dx.doi.org/10.1080/03093640600816982

13. Klodd E, Hansen AH, Fatone S, Edwards ML. Effects of prosthetic foot forefoot flexibility on gait of unilateral transtibial prosthesis users. J Rehabil Res Dev. 2010;47(9): 899-910. [PMID:21174254] http://dx.doi.org/10.1682/JRRD.2009.10.0166

14. Klodd E, Hansen AH, Fatone S, Edwards ML. Effects of prosthetic foot forefoot flexibility on oxygen cost and subjective preference rankings of unilateral transtibial prosthesis users. J Rehabil Res Dev. 2010;47(6):543-52.

[PMID:20848367]

http://dx.doi.org/10.1682/JRRD.2010.01.0003

15. Hansen AH, Childress DS, Knox EH. Prosthetic foot rollover shapes with implications for alignment of trans-tibial prostheses. Prosthet Orthot Int. 2000;24(3):205-15. [PMID:11195355] http://dx.doi.org/10.1080/03093640008726549

16. Lieber RL. Statistical significance and statistical power in hypothesis testing. J Orthop Res. 1990;8(2):304-9. [PMID:2303964] http://dx.doi.org/10.1002/jor.1100080221

17. Deathe AB, Miller WC. The L test of functional mobility: Measurement properties of a modified version of the timed “up \& go" test designed for people with lower-limb amputations. Phys Ther. 2005;85(7):626-35. [PMID:15982169]

18. Franchignoni F, Orlandini D, Ferriero G, Moscato TA. Reliability, validity, and responsiveness of the locomotor capabilities index in adults with lower-limb amputation undergoing prosthetic training. Arch Phys Med Rehabil. 2004;85(5):743-48. [PMID:15129398]

http://dx.doi.org/10.1016/j.apmr.2003.06.010

19. Quai TM, Brauer SG, Nitz JC. Somatosensation, circulation and stance balance in elderly dysvascular transtibial amputees. Clin Rehabil. 2005;19(6):668-76.

[PMID:16180604] http://dx.doi.org/10.1191/0269215505cr857oa 
JRRD, Volume 51, Number 1, 2014

20. Powell LE, Myers AM. The activities-specific balance confidence (ABC) scale. J Gerontol A Biol Sci Med Sci. 1995; 50A(1):M28-34. [PMID:7814786] http://dx.doi.org/10.1093/gerona/50A.1.M28

21. Miller WC, Speechley M, Deathe AB. Balance confidence among people with lower-limb amputations. Phys Ther. 2002;82(9):856-65. [PMID:12201800]

22. Hansen A, Sam M, Childress D. The effective foot length ratio (EFLR): A potential tool for characterization and evaluation of prosthetic feet. J Prosthet Orthot. 2004;16(2): 41-45.

http://dx.doi.org/10.1097/00008526-200404000-00002
Submitted for publication December 17, 2012. Accepted in revised form July 11, 2013.

This article and any supplemental material should be cited as follows:

Hansen A, Nickel E, Medvec J, Brielmaier S, Pike A, Weber M. Effects of a flat prosthetic foot rocker section on balance and mobility. J Rehabil Res Dev. 2014;51(1):13748.

http://dx.doi.org/10.1682/JRRD.2012.12.0229

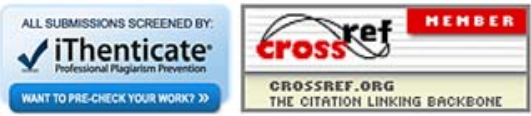

